Article

\title{
Reducing Friction with a Liquid Film on the Body Surface
}

\author{
Nikolay Klyuev ${ }^{1,2, *}$, Konstantin Polyakov ${ }^{1}$ and Alyona Krutovertseva ${ }^{2}$ \\ 1 Department of Mechanics, Samara State Technical University, Molodogvardeyskaya Street, Building 244, \\ Samara 443100, Russia; polyakov.ka@samgtu.ru \\ 2 Department of Aerogasdynamics and Heat Transfer, Joint Stock Company Space Rocket Centre Progress, \\ Zemetsa str., Building 18, Samara 443009, Russia; mail@samspace.ru \\ * Correspondence: Nikolay_klyuev@mail.ru; Tel.: +7-846-334-5440
}

Received: 23 January 2018; Accepted: 27 February 2018; Published: 7 March 2018

\begin{abstract}
A flow of a thin layer of liquid is simulated on a flat surface of a body located in a stream of air. Liquid film on the surface of the body reduces frictional resistance and can be used as a boundary layer control element. The paper presents a mathematical model of the film flow on a half-plane, located at an angle to the horizon. The fluid flow is determined by the force of gravity and friction from the external air current. A model of an incompressible viscous fluid is used in the boundary-layer approximation. The terms of the motion equation are averaged over the film thickness according to the Leibniz rule. In the cross section of the film, a quadratic law is adopted for the distribution of the longitudinal velocity, taking into account friction on the film surface. An analytical solution of the problem is obtained in the form of series in powers of the small parameter for determining the film thickness and the average longitudinal velocity along the length of the plate. It is shown that the friction decreases with flow around a half-plane with a film of liquid on the surface.
\end{abstract}

Keywords: film; liquid; boundary layer; current; friction; small parameter

\section{Introduction}

Problems of liquid film flow are quite often encountered in technical applications and are discussed by many authors. Paper [1] presents the results of investigation of films for a gas-liquid two-phase flow in short horizontal pipes. The obtained results are compared with the experimental data. In paper [2], the dynamic characteristics of the film flow with a counter-flow of air are given. The calculated data is experimentally confirmed.

A three-dimensional dynamic model of film flow on a cylindrical surface is considered in paper [3]. The film is affected by force of gravity and surface tension, as well as the strength of resistance from the counter flow of gas. In paper [4], the solution of the conjugate problem for the air flow over a flat plate with a thin liquid film on the surface is presented. The film is formed by extruding liquid from a porous wall. In paper [5], the film is formed from the external medium. A mathematical model is obtained for the zero approximation. The results of theoretical and numerical studies of liquid films are presented in papers [6,7], and experimental studies have been performed in [8-10].

A model of a thin film flow along an inclined plane is presented in paper [11]. The terms of Navier-Stokes equations and the energy equations are averaged over the depth of the liquid. A mathematical model of thin film evaporation was proposed in paper [12]. In paper [13], experimental results and numerical modeling of the night flow on a cooled wall are presented. The measurements were carried out on a flat plate. The problem of condensate film stability on a vertical surface is analytically studied in paper [14]. An analytical model for the laminar flow of a condensate film on the outer surface of an isothermal vertical pipe is presented in [15]. 
Despite the large number of works on the flow of liquid films, there is still a considerable interest in this phenomenon. Liquid film on the surface of the body reduces friction resistance and can be used as a control element for the boundary layer. In this study, the flow of a thin layer of liquid on a flat surface of the body in the airflow is simulated for its ability to reduce frictional resistance.

\section{Materials and Methods}

As an element of the surface, we choose a plane and consider the steady-state flow of a liquid film under the action of gravity and the incoming air flow (Figure 1). The film is formed by squeezing the liquid through the porous surface of the plate, which is located at $\alpha$ angle to the horizon. The liquid flows evenly into the film at $V_{k}$ speed.

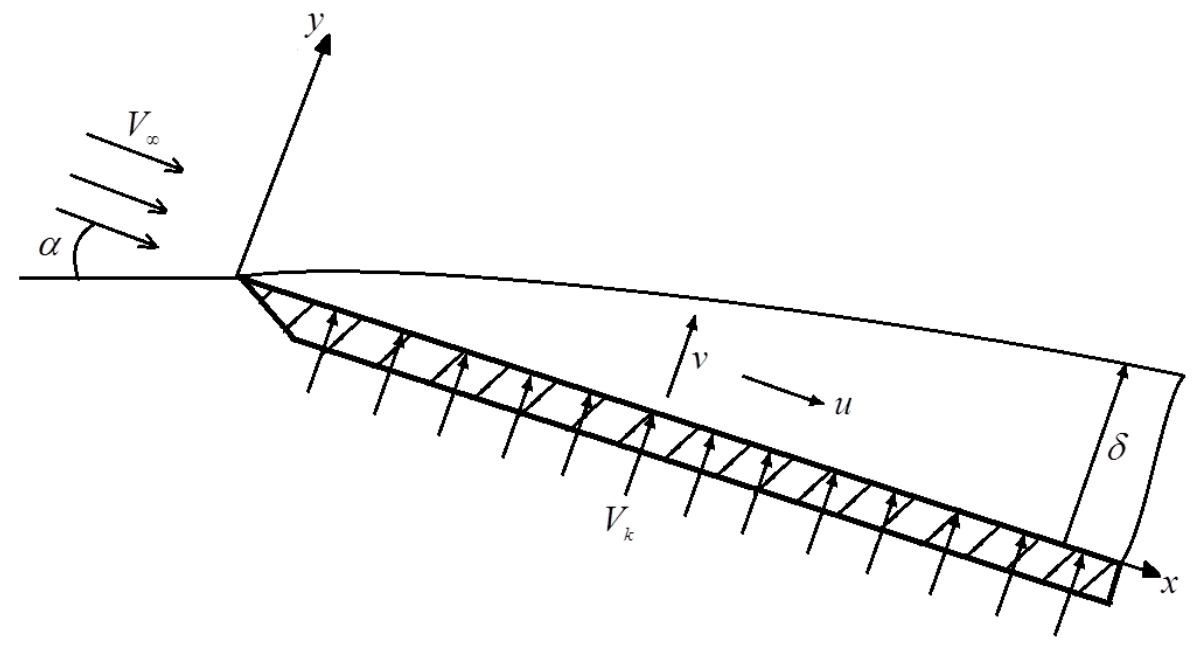

Figure 1. Flow pattern of the film: $u, v$-components of the velocity vector, $V_{k}$-rate of fluid flow, $V_{\infty}$-speed of the incoming flow, $\delta$-film thickness.

In the boundary-layer approximation, the mathematical formulation of the problem has the form

$$
\begin{gathered}
\frac{\partial u}{\partial x} u+\frac{\partial u}{\partial y} v=g \sin \alpha+v \frac{\partial^{2} u}{\partial y^{2}} \\
\frac{\partial p}{\partial y}=0, \quad \frac{\partial u}{\partial x}+\frac{\partial v}{\partial y}=0 .
\end{gathered}
$$

The boundary conditions of the problem will have the following form

$$
\begin{gathered}
x=0, y=0, u=v=0, \\
x>0, y=0, u=0, v=v_{k}, y=\delta, v=v(\delta), \frac{\partial u}{\partial y}=\frac{\tau}{\mu},
\end{gathered}
$$

where $p$-pressure, $\rho$-density, $v, \mu$-kinematic and dynamic viscosity, $g$-acceleration of gravity, $\tau$-tangential stress at the boundary between the film - the incoming air flow.

\section{Methods of Solution}

Combining the equation of motion (8) with the continuity equation, obtain the conservative form

$$
\frac{\partial u^{2}}{\partial x}+\frac{\partial(u v)}{\partial y}=g \sin \alpha+v_{3} \frac{\partial^{2} u}{\partial y^{2}}
$$

We carry out the averaging of the terms entering into Equation (5) over the thickness of the film. 
For this purpose, we integrate (10) with respect to $y$ from 0 to $\delta$, using the Leibniz rule (derived from an integral with a variable upper limit)

$$
\frac{d}{d t} \int_{0}^{\delta} u d y=u(\delta) \frac{\partial \delta}{\partial t}+\int_{0}^{\delta} \frac{\partial u}{\partial t} d y,
$$

We use the definition of the mean longitudinal flow velocity

$$
<u>=\frac{1}{\delta} \int_{0}^{\delta} u(x, y) d y .
$$

The integral of the first term of Equation (5) is written in the form (the complete derivative can be replaced by a partial one)

$$
\int_{0}^{\delta} \frac{\partial u^{2}}{\partial x} d y=\frac{\partial}{\partial x} \int_{0}^{\delta} u^{2} d y-u^{2}(\delta) \frac{\partial \delta}{\partial x} .
$$

Since the thickness of the film is sufficiently small, the viscous forces will play a decisive role in the formation of the flow. In this case, we can take for the longitudinal velocity the quadratic law of variation of the longitudinal velocity in the cross section of the film. We use the expression for the velocity of a film of constant thickness that flows along a flat vertical wall, taking into account friction on the surface

$$
u(y)=\frac{g \sin \alpha \rho \delta^{2}}{\mu}\left[\frac{y}{\delta}-\frac{1}{2}\left(\frac{y}{\delta}\right)^{2}\right]+\frac{y \tau}{\mu} .
$$

Average flow velocity

$$
<u>=\frac{g \sin \alpha \rho \delta^{2}}{3 \mu}+\frac{\delta \tau}{2 \mu} .
$$

Taking into account (10), the longitudinal velocity (9) can be rewritten

$$
u(y)=3<u>\left[\frac{y}{\delta}-\frac{1}{2}\left(\frac{y}{\delta}\right)^{2}\right]+\frac{\tau}{2 \mu_{3}}\left(\frac{3 y^{2}}{2 \delta}-y\right),
$$

For the problem under consideration, the average flow velocity $\langle u\rangle$, the friction on the surface of the film $\tau$, and the thickness of the film $\delta$ are functions of the coordinate $\mathrm{x}$.

Using (11) we write the following expressions

$$
\begin{gathered}
u(\delta)=\frac{3<u>}{2}+\frac{\delta \tau}{4 \mu}, \frac{\partial u(\delta)}{\partial x}=\frac{3}{2} \frac{\partial<u>}{\partial x}+\frac{\tau}{4 \mu} \frac{\partial \delta}{\partial x}, \\
u^{2}(y)=9<u>^{2}\left[\frac{y^{2}}{\delta^{2}}-\frac{y^{3}}{\delta^{3}}+\frac{y^{4}}{4 \delta^{4}}\right]+\frac{3 \tau<u>}{\mu}\left(\frac{2 y^{3}}{\delta^{2}}-\frac{3 y^{4}}{4 \delta^{3}}-\frac{y^{2}}{\delta}\right)+\frac{\tau^{2}}{4 \mu^{2}}\left(\frac{3 y^{2}}{2 \delta}-y\right)^{2}, \\
u^{2}(\delta)=\frac{1}{4}\left(9<u>^{2}+\frac{3 \delta \tau<u>}{\mu}+\frac{\delta^{2} \tau^{2}}{4 \mu^{2}}\right), \int_{0}^{\delta} u^{2} d y=\frac{6}{5}<u>^{2} \delta+\frac{\delta^{2} \tau<u>}{20 \mu}+\frac{\delta^{3} \tau^{2}}{120 \mu^{2}} \\
\frac{\partial u}{\partial y}=3<u>\left(\frac{1}{\delta}-\frac{y}{\delta^{2}}\right)+\frac{\tau}{2 \mu}\left(\frac{3 y}{\delta}-1\right),\left(\frac{\partial u}{\partial y}\right)_{y=0}=\frac{3<u>}{\delta}-\frac{\tau}{2 \mu},\left(\frac{\partial u}{\partial y}\right)_{y=\delta}=\frac{\tau}{\mu}
\end{gathered}
$$

Using the obtained expressions (11)-(15), we rewrite the equation of motion in the following form

$$
\begin{gathered}
\frac{1}{5} \delta \frac{\partial<u>}{\partial x}(12<u> \\
\left.+\frac{1}{4 \mu} \tau \delta\right)-\left(\frac{21}{20}<u>^{2}+\frac{13}{20 \mu} \tau \delta<u>+\frac{3}{80 \mu^{2}} \tau^{2} \delta^{2}\right) \frac{\partial \delta}{\partial x} \\
+u(\delta) v(\delta)=g \delta \sin \alpha+\frac{3 \tau}{2 \rho}-\frac{3 v<u>}{\delta} .
\end{gathered}
$$


We write down the law of conservation of mass, for which we integrate the continuity Equation (2) with $y$ from 0 to $\delta$

$$
v(\delta)=v_{k}-\frac{\partial u}{\partial x} \delta+\frac{u}{2} \frac{\partial \delta}{\partial x}+\frac{\tau \delta}{4 \mu} \frac{\partial \delta}{\partial x} .
$$

Taking into account expressions (12) and (17), Equation (16) can be rewritten

$$
\begin{aligned}
\delta \frac{\partial u}{\partial x}\left(\frac{9}{10} u-\frac{3}{10 \mu} \tau \delta\right)- & \left(\frac{3}{10} u^{2}-\frac{1}{40 \mu^{2}} \tau^{2} \delta^{2}+\frac{3}{20 \mu} \tau \delta u\right) \frac{\partial \delta}{\partial x}+\left(\frac{3 u}{2}+\frac{\delta \tau}{4 \mu}\right) v_{k} \\
& =\delta g \sin \alpha+\frac{3}{2 \rho} \tau-3 v \frac{u}{\delta}
\end{aligned}
$$

If we exclude a neighborhood of a point $x=0$, then $v(\delta)<<v_{k}$, in the first approximation, the continuity equation takes the form

$$
v_{k}-\frac{\partial u}{\partial x} \delta+\frac{u}{2} \frac{\partial \delta}{\partial x}+\frac{\tau \delta}{4 \mu} \frac{\partial \delta}{\partial x}=0
$$

We introduce dimensionless variables

$$
\xi=\frac{v_{k}}{v} \sqrt[3]{\frac{6 v v_{k}}{g} x},<u>=\left(\frac{v}{v_{k}}\right)^{2} \frac{g}{3 v} U(\xi), \delta=\frac{v}{v_{k}} \Delta(\xi), \tau=\frac{\mu g}{3 v_{k}} T,
$$

and, taking into account (20), Equations (18) and (19) take the form

$$
\begin{gathered}
\Delta^{2}(3 U-\Delta T) \frac{d U}{d \xi}-\Delta\left(U^{2}-\frac{\Delta U T}{2}-\frac{\Delta^{2} T^{2}}{12}\right) \frac{d \Delta}{d \xi}-15 \xi^{2}\left(\Delta^{2} \sin \alpha-U-\frac{\Delta U}{2}+\frac{T \Delta}{2}-\frac{T \Delta^{2}}{12}\right)=0 \\
2 \Delta \frac{d U}{d \xi}-U \frac{d \Delta}{d \xi}-3 \xi^{2}-\frac{1}{2} T \Delta \frac{d \Delta}{d \xi}=0
\end{gathered}
$$

initial conditions of the task

$$
\xi=0: \quad U=\Delta=0 .
$$

Calculation of friction is feasible according to the approximate model. The film thickness is sufficiently small and it can be neglected, then the friction value is determined from the Blasius problem

$$
\tau_{w}(x)=0.332 \sqrt{\frac{\mu \rho U_{\infty}^{3}}{x}} .
$$

For the speed of the oncoming stream $U_{\infty}=5 \mathrm{~m} / \mathrm{s}, v_{k}=8 \times 10^{-4} \mathrm{~m} / \mathrm{s}$ and the length of the wall $l=0.5 \mathrm{~m}$. Reynolds number $R e=1.68 \times 10^{5}$, which corresponds to laminar flow. If the injection of the mass is carried out by slightly receding from the edge of the plate, then the distribution of friction on the surface of the film can be considered a constant value and take its average value

$$
\tau=\frac{1}{0.5} \int_{0}^{0.5} \tau_{w}(x) d x
$$

Moving to dimensionless quantities, we obtain $T=0.67=$ const.

Note that for the initial data (liquid-water at temperature $t=20^{\circ} \mathrm{C}$ ), $v=1.01 \times 10^{-6} \mathrm{~m}^{2} / \mathrm{s}$, $\rho=998 \mathrm{~kg} / \mathrm{m}^{3}$ dimensionless coordinate is a small quantity $0<\xi<1.2 \times 10^{-2}$, that is $\xi<<1$.

Consequently, the solution of Equations (21) and (22) can be represented in the form of series in powers of the small parameter

$$
U=\sum_{m=1}^{\infty} \alpha_{m} \xi^{m} \text { and } \Delta=\sum_{m=1}^{\infty} \beta_{m} \xi^{m}
$$


The coefficients $\alpha_{m}$ and $\beta_{m}$ are determined by substituting the expansions (24) into (21) and (22) and equating the coefficients to zero for identical powers of $\xi$.

\section{Results and Discussions}

For $\alpha=\pi / 6$ we get

$$
U=1.105 \xi^{2}-0.225 \xi^{3}+\ldots \Delta=1.197 \xi+0.805 \xi^{2}+\ldots
$$

for $\alpha=\pi / 2$ the solution takes the form

$$
U=1.956 \xi^{2}-0.514 \xi^{3}+\ldots \Delta=1.224 \xi+0.963 \xi^{2}+\ldots
$$

Graphs (Figures 2-4) present comparative flow characteristics, where $1-\alpha=\pi / 2,2-\alpha=\pi / 6$.

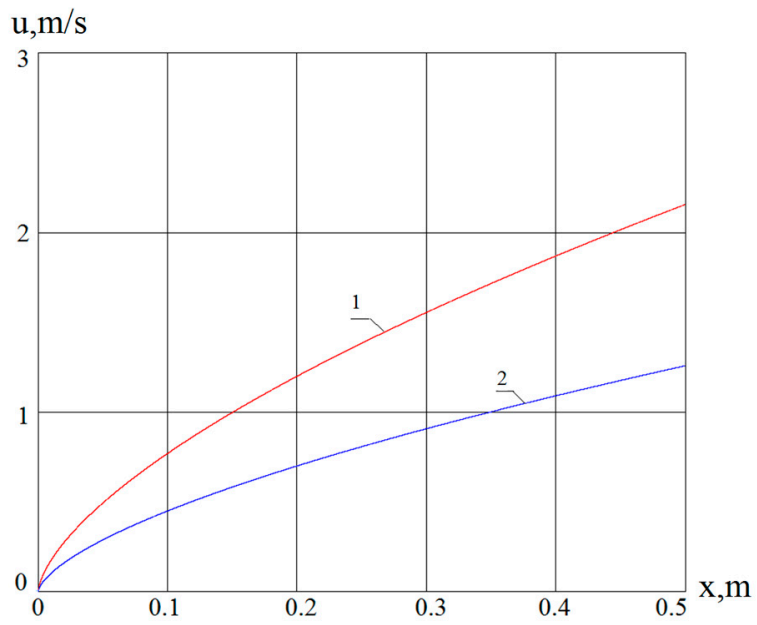

Figure 2. The average longitudinal velocity.

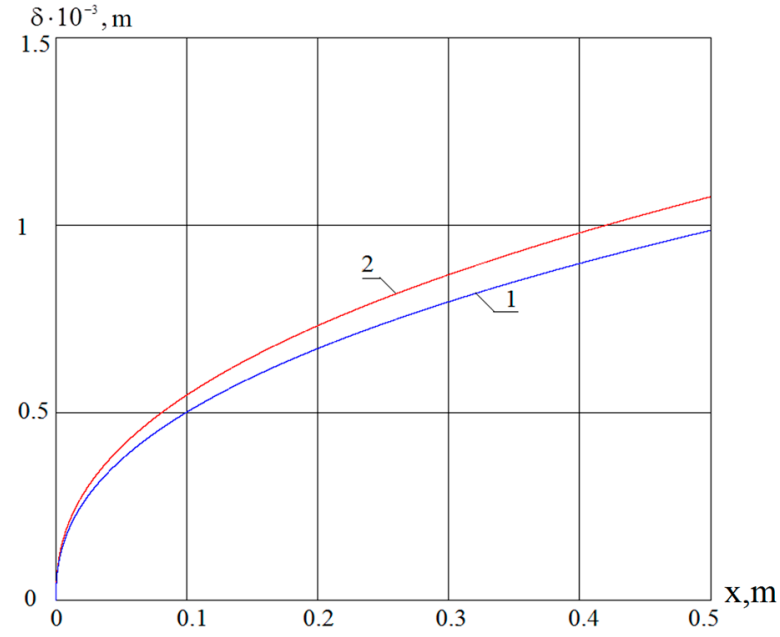

Figure 3. Film thickness. 


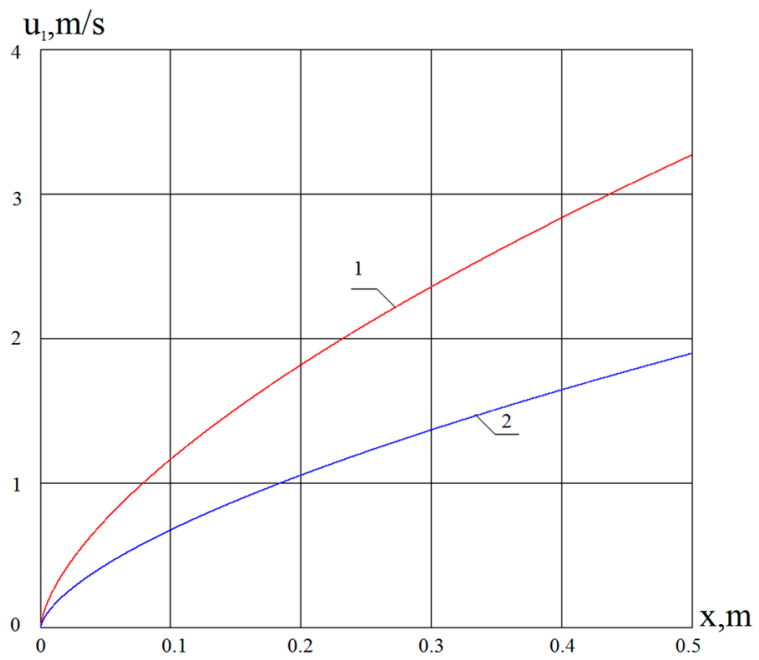

Figure 4. The fluid velocity at film surface.

Then we consider air stream flow by a flat plate (the Blasius problem), but with a movable wall

$$
\frac{\partial u}{\partial x} u+\frac{\partial u}{\partial y} v=v \frac{\partial^{2} u}{\partial y^{2}}, \frac{\partial p}{\partial y}=0, \frac{\partial u}{\partial x}+\frac{\partial v}{\partial y}=0
$$

boundary conditions

$$
y=0, u=u_{0}, v=0, y=\infty, u=u_{\infty}
$$

Such a formulation allows a self-similar solution for $u_{0}=$ const (for $u_{0}=f(x)$ the problem it is unjustifiably more complicated).

Let us calculate the average velocities on the surface of the film (for $\alpha=\pi / 2, u_{0}=2 \mathrm{~m} / \mathrm{s}$; $\alpha=\pi / 6, u_{0}=1.16 \mathrm{~m} / \mathrm{s}$ ) and perform the numerical solution of problem (27).

The results are shown in the graph (Figure 5) in the form of friction distribution on the plate surface, where curve 1 , as before, denotes the slope angle $\alpha=\pi / 2$, and distribution 3 is the solution of the classical Blasius problem.

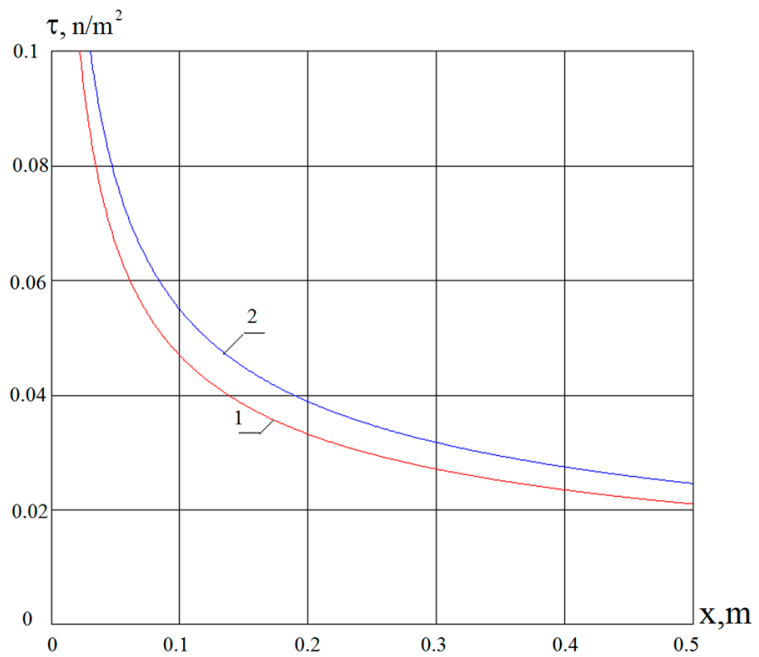

Figure 5. Tangential stress on the inter-phase surface.

For the slope of the plate $\alpha=\pi / 6$, the results are between 1 and 3 . The friction reduction, calculated from the average value, is $14.6 \%$. 


\section{Conclusions}

An analytical solution of the problem is obtained for the average velocity and film thickness in the form of series in powers of the small parameter with allowance for the zero and first approximations. Dependency curves are constructed for the film thickness and the average longitudinal velocity on the length of the plate. The calculation of friction is based on an approximate model. The friction is reduced with the air flow around the plate. The obtained results can be used to calculate the frictional resistance, which is a component of the aerodynamic drag of an aircraft.

Author Contributions: Nikolay Klyuev developed a mathematical model and wrote the methods and solutions section and conclusions section. Konstantin Polyakov performed numerical calculations, wrote the Results and discussion section and translated the article into English. Alyona Krutovertseva found scientific articles on the subject of research and wrote an introduction and references section.

Conflicts of Interest: The authors declare no conflict of interest. The founding sponsors had no role in the design of the study; in the collection, analyses, or interpretation of data; in the writing of the manuscript, and in the decision to publish the results.

\section{References}

1. López, J.; Pineda, H.; Bello, D.; Ratkovich, N. Study of liquid-gas two-phase flow in horizontal pipes using high speed filming and computational fluid dynamics. Exp. Therm. Fluid Sci. 2016, 76, 126-134. [CrossRef]

2. Lu, H.; Lu, L.; Luo, Y.; Qi, R. Investigation on the dynamic characteristics of the counter-current flow for liquid desiccant dehumidification. Energy 2016, 101, 229-238. [CrossRef]

3. Li, M.; Lu, Y.; Zhang, S.; Xiao, Y. A numerical study of effects of counter-current gas flow rate on local hydrodynamic characteristics of falling films over horizontal tubes. Desalination 2016, 383, 68-80. [CrossRef]

4. Klyuev, N.I.; Gimadiev, A.G.; Kryukov, Y.A. Two-media boundary layer on a flat plate. Int. J. Eng. Technol. 2014, 6, 2368-2374.

5. Klyuev, N.I.; Kryukov, Y.A. Influence of fluid film on friction of a flat plate. Russ. Aeronaut. (Iz. VUZ) 2014, 57, 372-377. [CrossRef]

6. Camassa, R.; Ogrosky, H.R. On viscous film flows coating the interior of a tube: Thin-film and long-wave models. J. Fluid Mech. 2015, 772, 569-599. [CrossRef]

7. Muramatsu, K.; Youn, Y.; Han, Y.; Hasegawa, Y.; Shikazono, N. Numerical study on the effect of initial flow velocity on liquid film thickness of accelerated slug flow in a micro. Int. J. Heat Fluid Flow 2015, 54, 77-86. [CrossRef]

8. Han, Y.; Kanno, H.; Ahn, Y.-J.; Shikazono, N. Measurement of liquid film thickness in micro tube annular flow. Int. J. Multiph. Flow 2015, 73, 264-274. [CrossRef]

9. Youn, Y.J.; Muramatsu, K.; Han, Y.; Shikazono, N. The effect of initial flow velocity on the liquid film thickness in micro tube accelerated slug flow. Int. J. Multiph. Flow 2015, 73, 108-117. [CrossRef]

10. Ju, P.; Brooks, C.S.; Ishii, M.; Liu, Y.; Hibiki, T. Film thickness of vertical upward cocurrent adiabatic flow in pipes. Int. J. Heat Mass Transf. 2015, 89, 985-995. [CrossRef]

11. Richard, G.L.; Ruyer-Quil, C.; Vila, J.P. A three-equation model for thin films down an inclined plane. J. Fluid Mech. 2016, 804, 162-200. [CrossRef]

12. Akkuş, Y.; Dursunkaya, Z. A new approach to thin film evaporation modeling. Int. J. Heat Mass Transf. 2016, 101, 742-748. [CrossRef]

13. Liu, C.L.; Liu, J.L.; Zhu, H.R.; Wu, A.S.; He, Y.H.; Zhou, Z.X. Film cooling sensitivity of laidback fanshape holes to variations in exit configuration and mainstream turbulence intensity. Int. J. Heat Mass Transf. 2015, 89, 1141-1154. [CrossRef]

14. Degan, G.; Sanya, A.; Akowanou, C. Laminar film condensation along a vertical plate embedded in an anisotropic porous medium with oblique principal axes. Heat Mass Transf. 2016, 52, 2119-2128. [CrossRef]

15. Kim, S.; Lee, Y.G.; Jerng, D.W. Laminar film condensation of saturated vapor on an isothermal vertical cylinder. Int. J. Heat Mass Transf. 2015, 83, 545-551. [CrossRef]

(C) 2018 by the authors. Licensee MDPI, Basel, Switzerland. This article is an open access article distributed under the terms and conditions of the Creative Commons Attribution (CC BY) license (http://creativecommons.org/licenses/by/4.0/). 